\title{
Medical Multimedia Retrieval 2.0
}

\author{
H. Müller \\ Medical Informatics, University and Hospitals of Geneva \\ and Business Information Systems, University of Applied Sciences, Sierre, Switzerland
}

\section{Introduction}

Medical multimedia retrieval has been an extremely active research domain over the past 10 years [1], and the pace of research is still increasing. In nonmedical fields a correspondingly strong research thrust had started already in the early 1990s [2,3]. The availability of visual data in digital format and the necessary computing power to analyse the data automatically led to great enthusiasm for the potential of multimedia retrieval and a considerable degree of hype. A driving force behind visual information retrieval was the inability to search without textual annotations for meaningful results in large databases of visual information that contain often only little textual annotation or structure.

In the medical domain visual information retrieval was proposed many times in the late 1990s [4,5]. Real applications in clinical practice with large databases remained rare, also related to the fact that most hospitals became fully digital only over the past few years [6]. In the past ten years research projects such as Assert [7], IRMA ${ }^{1}$ (Image Retrieval in Medical Applications [8]), and MedGIFT $^{2}$ (Medical GNU Image Finding Tool, [9]) have advanced the field through a fairly large number of publications and explorations of several sub-domains of medical image re-

http://www.irma-project.org/

http://www.sim.hcuge.ch/medgift/ trieval such as varying feature spaces, interaction with the user [10], evaluation of real user needs [11], and evaluations of image retrieval systems [12]. A first study in clinical practice showed that the diagnostic performance of participating clinicians improved significantly with the use of an image retrieval system, this being particularly noticeable for less experienced clinicians [7]. The type of images and the specific application domains in medical imaging vary very strongly ranging from radiology to histopathology [13] and many other specialties. In general, much of the medical image and signal processing research was frequently disconnected from clinical practice. Image retrieval by its applied nature can help to bring the theoretical research closer to the clinicians but clear use cases are necessary and domains where such applications can have an important impact such as the retrieval of lung images by texture $[10,14]$ or the retrieval of similar fractures for operation planning [15]. While such clear use cases are necessary for image retrieval to become a success in the medical field, this article rather focuses on the potential influence of Web 2.0 techniques on medical visual information retrieval. The motivation for this article is to review the very recent literature in the medical imaging and medical visual information retrieval field to identify trends and missing links. It particularly tries to link the fields of Web 2.0 and visual information retrieval to identify important trends and missing components. 
The environment of the medical patient record has changed enormously over the past ten years, going from mainly paper- and film-based records to the electronic record and now to the integrated multimedia patient record [16]. This has created an enormous growth in the quantity of data produced, including large amounts of medical images and increasingly videos from cardiology, ultrasound, endoscopy, and sleep laboratories to just name a few sources. In Geneva University Hospitals, the Radiology Department alone produced over 70,000 images per day in 2007, mainly through multi-slice tomographic series. At the same time the complexity of data is growing commensurably, as an increasing number of diagnostic procedures and exams are developed that are becoming increasingly complex (varying procedures, contrast agents, slice thicknesses in tomographic data, etc.). Examples for this rising complexity are also radiation therapy procedures [17] or when trying to estimate radiation doses for patients in common exams [18]. Through the electronic patient record all these data are available to any treating clinician, in contrast to earlier practice, where they were typically only seen by specialists (as in radiology), who then wrote a textual description of the main findings. To avoid data overload for non-specialists, new tools are required to help clinicians view and interpret the large amount of information that is available. These tools often and increasingly have to include visual data for which interpretation is frequently complex. A particularly promising direction for visual analysis is to search for similar cases taking into account visual, textual, and structured information on patients [19]. Still, many legal issues need to be solved as currently in most countries the use of patient data is restricted to the treatment of a single patient and even anonymous use of other related patients' data is often hard or impossible to obtain because of difficulties in getting approval for access. The American Medical Informatics Association (AMIA) has already identified this need and created a working group and a meeting to work on rules for such secondary use of patient data in anonymised form $[20,21]$. Considerable economical benefits are expected [22], as much knowledge is stored in longitudinal collections of patient care visits, and making these experiences available without compromising the privacy of the patients can be a very valuable aide for knowledge discovery. Data exchanges across institution boundaries are also beginning [23] and are likely to even further increase the need to develop tools to manage these large amounts of patient data. The goal of such inter-institutional data exchanges is to make available all data on a patient wherever it is stored. Currently, most patient data are scattered around many institutions and practitioners, sometimes in a particular region, but often even more widely diffused. Combining all these data can potentially help to detect unknown diseases, improve patient care and at the same time lower healthcare costs. Learning from past cases for future treatments also becomes increasingly interesting if more cases are available for analysis.

A clear definition of what Web 2.0 exactly means has not yet emerged, but there are many concepts unique to Web 2.0 that can be mentioned in this context [24]. Early Web 2.0 technologies included, for example AJAX (Asynchronous Javascript and XML) and web services that allowed developing user interfaces within a web browser that could rival the usability of "normal" or desktop user interfaces regarding speed and comfort. Based on these technologies users were increasingly in- cluded as active content providers and not only passive content users as was the case with Web 1.0.

This article cannot give a complete overview of Web 2.0 applications and trends, so only a few are mentioned, grouped into the following categories:

- Wikis, collaborative development of documents (Wikipedia ${ }^{3}$ );

- Blogs and podcasts, allowing all users to easily express themselves and publish through text and/or sound and video (BlogSearchEngine ${ }^{4}$ );

- APIs (Application Programming Interfaces) for many applications to reuse a particular functionality in one's own web pages or applications $\left(\right.$ Google Earth ${ }^{5}$;

- Simple tools to work in collaborations, such as organising joint meetings (Doodle ${ }^{6}$ );

- Personal sites to promote oneself, share information on oneself and contacts with others, (LinkedIn ${ }^{7}$, Facebook $^{8}, \ldots$ );

- Community-building around certain topics (del.icio.us ${ }^{9}$ on bookmarks, youTube ${ }^{10}$ on videos, FlickR ${ }^{11}$ on images);

- 3D communities modelled similar to the real world (Secondlife ${ }^{12}$ ).

There has been considerable criticism about many of these techniques, such as those used in Wikipedia, where the quality is often not judged to be high enough, since anyone can modify the content. Studies, however, show fairly good quality compared to printed encyclopaedias [25]. Of course, there is little control in many blogs, and the users need to know the specific field to actually be able to

\footnotetext{
http://www.wikipedia.org/

http://www.blogsearchengine.com/

http://earth.google.com/

http://www.doodle.com/

http://www.linkedin.com/

http://www.facebook.com/

http://del.icio.us/

http://www.youtube.com/

http://www.flickr.com/

http://www.secondlife.com/
} 
judge the information presented and hopefully discriminate the good from the bad. Still, the success of Web 2.0 shows that by creating incentives for participation, added value can be created for users within specific communities. YouTube is already responsible for more than $10 \%$ of Internet traffic after only two years of existence, underlining the phenomenal growth in this domain.

In the next section, methods will be described for obtaining the references and data used in this article, followed by our results ranked according to the influence of Web 2.0 on the medical field in general, and then on recent developments in the medical multimedia retrieval field.

\section{Methods}

This article is mainly based on a literature search using the Pubmed ${ }^{13}$ search engine and the Google Scholar ${ }^{14}$ search engine. First, all articles published in the year 2007 in 12 journals linked with medical imaging were reviewed for choosing the best articles for the Yearbook of Medical Informatics. The selection criteria were the importance of the technology on medical informatics, the novelty of the article in the domain of medical imaging, and the quality of the article itself (literature review, description of the techniques, evaluation). In addition, and so as to focus the article on visual information, retrieval searches were performed with the search terms "medical image retrieval" and "medical multimedia retrieval". The search was not exhaustive, but rather the first 200 results were taken into account, placing more weight on recent articles to cover the very recent state of

$13 \mathrm{http}: / /$ www.pubmed.gov/

$14 \mathrm{http}: / /$ scholar.google.com/ the art. The main goal of this search was to obtain an overview of current trends in medical multimedia retrieval and map them on current developments in medical imaging and the Web 2.0 field. The selection of the references cited in this article was based on their novelty in image retrieval, their scientific validity and the link to the subject of Web 2.0. Many of the highly ranked articles in Google Scholar were several years old. After the first 200, the results increasingly include variations of already reviewed articles, or irrelevant articles. In Pubmed the results are ordered by date of publication and after the top 200 results one similarly notices that the articles become either older or less focused on image retrieval. To get a clearer idea of the field in general, a web search was also performed directly in Google mixing search terms of "medical image retrieval" and "medicine" with Web 2.0 terms such as "Web 2.0", "Wiki", "Blog", etc. The articles and web reports regarded as most important are presented in the following sections based mainly on their novelty.

\section{Results}

This section describes the main results of the literature search. First, Web 2.0 techniques in the medical field are presented and then their direct influence on multimedia retrieval are highlighted. The last subsection describes those techniques that have not yet been used in the multimedia retrieval field. These techniques might be interesting for future developments in medical visual information retrieval.

\section{Web 2.0 in the Medical Field}

The influence of the Internet on the medical field has been studied for a very long time and accreditation systems for web pages with medical content exist such as the $\mathrm{HON}^{15}$ (health on the net) code [26]. Still, most of the current web pages with medical content such as MedlinePlus ${ }^{16}$ rather see the user as a consumer and not really as a content provider or sharer, although many interactive tutorials exist to obtain a maximum of information from the users in an interactive manner and explain fields such as post traumatic stress disorders (see Figure 1). This is only partly Web 2.0 as it provides a user interface similar to desktop applications and allows the user to personalize the results by entering information and not only consuming it.

Trust is the most common question linked to medical content on the Internet and is also crucial for the functioning of Web 2.0. In one extreme, Google has been tested directly as a diagnostic aid tool, which might sound scary, but results seem to be promising [27]. It has to be recognized that such behaviour, of searching on Google for important answers is a current reality and quite frequently used when other high quality information sources might not be accessible, this can be a second solution to obtain support for or against a decision. In the clinical field this tends to happen when no other knowledge sources of comparable or better quality are available to clinicians. In [28], a more general description of the Web 2.0 influence on the medical field is given, highlighting the need to create thematic networks among clinicians to exchange information and create a trust relationship among the participants. This moves away from the consumer model and towards a participative approach not

\footnotetext{
5 http://www.hon.ch/

16 http://medlineplus.gov/
} 


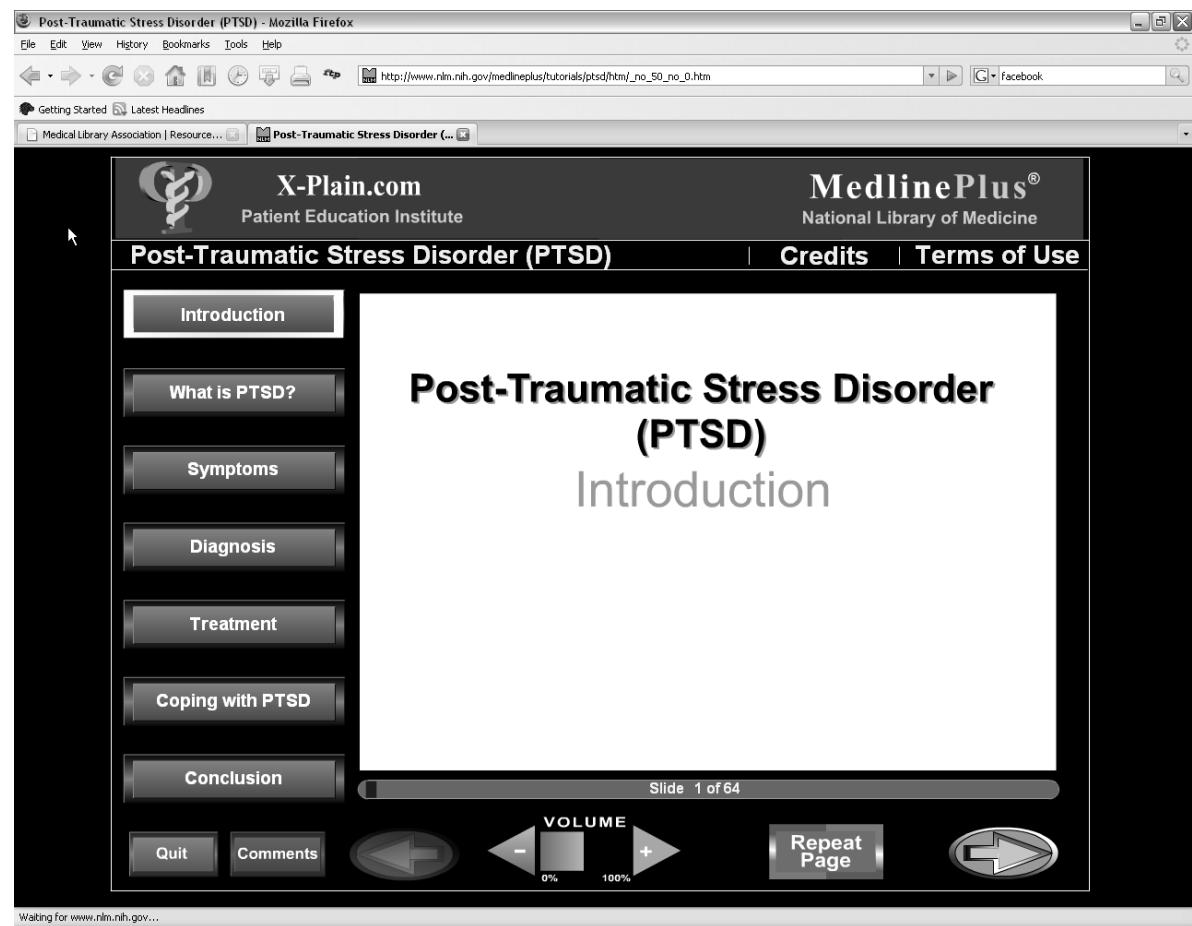

Fig. 1 An interactive web tutorial from the MedlinePlus Web page showing the multimedia capacities of modern web pages and the value that can add to enter information and develop more interactive web-based applications.

limiting such networks to clinicians but expanded to include all stakeholders in the health field for sharing their particular knowledge. One of the goals mentioned in the article is the free sharing of knowledge including knowledge stored in scientific articles to be made available. This can also limit the need for expensive knowledge repositories such as UpToDate ${ }^{17}$, where selected authors provide regularly updated overview articles for many topics.

Many of the concepts of Web 2.0 described above do exist in the medical field as well. Wikipedia has many medical topics and is particularly quick in updating data and providing links to the most important web pages, for example on epidemics such as SARS (Severe Acute Respiratory Syndrome) or West Nile Fever. Fully medical Wikis exist

17 http://www.uptodate.com/ as well, such as AskDrWiki ${ }^{18}$, which is a not-for-profit association for sharing medical knowledge. Read-only access is possible for everyone but a registration and acceptance is necessary for write access. This also adds a level of quality control to the content creation process.

Medical Blogs include "Clinical Cases and Images" 19 with images being directly available via FlickR, another Web 2.0 icon. Many medical subjects are covered, and a vast amount of other information is linked with these cases. A large number of blogs and podcasts are also described in the following article [29].

RSS feeds in the medical field equally exist in very large numbers to obtain updated knowledge on certain topics,

\footnotetext{
18 http://www.askdrwiki.com/

19 http://casesblog.blogspot.com/
}

as for example the selection linked from Medicinenet ${ }^{20}$.

An even more innovative approach of using Web 2.0 technologies in the medical field is described in [30], where an API similar to Google earth is used. This API maps the entire human body and makes it available in a 3D model. From medical texts in the electronic patient record it then tries to extract anatomical information to map these documents onto the 3D body map. The goal of this is to acquire information in complex documents and texts quicker by knowing exactly, which anatomical region the document is linked to. First tests seem very positive and integration into other clinical applications seems possible [30].

This article should be taken as a starting point for exploring this field as it is far from comprehensive in its coverage of the many articles and web pages dealing with medical topics in Web 2.0. What we cite supply further links for reading and discovery.

\section{Influence of These Technologies on Recent Medical Multimedia Retrieval}

Medical multimedia retrieval has long been a purely technology-driven and influenced domain, where small datasets were used to evaluate a technology that often existed without a user interface. Main advances in the field concentrated on the visual features being used to describe the images. Even today, research on defining and extracting visual features involves many new technological advances for visual information retrieval that need to be taken into account [31].

First trends in using Web 2.0 were the user interfaces that became increasingly

${ }^{20}$ http://www.medcicinnet.com/rss/article.htm 
web-based not only for image retrieval but even for access to an entire PACS system [32]. This allows for easy utilization across many platforms (Windows, MacOS, Unix) and eliminates the problem in many institutions that additional software cannot be installed on clinical computers due to security and confidentiality considerations (and most image retrieval systems are not standard clinical applications but only research prototypes not integrated into any clinical architecture). Interfaces also look increasingly like desktop applications as can be seen in Figure 2. This application is also described in [14]. It allows for a 3D annotation of regions of interest in tomographic lung images to classify the tissue in these regions and search for other cases with visually similar regions.

As these retrieval applications are Webbased they can easily be linked with other applications such as medical teaching files [33] that were in many institutions the first collaborative imaging applications allowing many clinicians to share images in medical cases, mainly for teaching. In the University of Geneva Radiology Department, at first many people were reluctant to share their data on such a common platform but once they realised that they too could profit from the wider availability of these images, they started sharing images in increasing numbers. The current teaching file Casimage ${ }^{21}$ [33] has over 80,000 images stored in the institution, with a subset of these being available on the Internet.

The sharing of medical imaging data within a community is definitely the area where medical image retrieval has made the greatest progress over the past years. The limited availability of reference image datasets in medical im-

\footnotetext{
${ }_{21}$ http://pubimage.hcuge.ch/
}

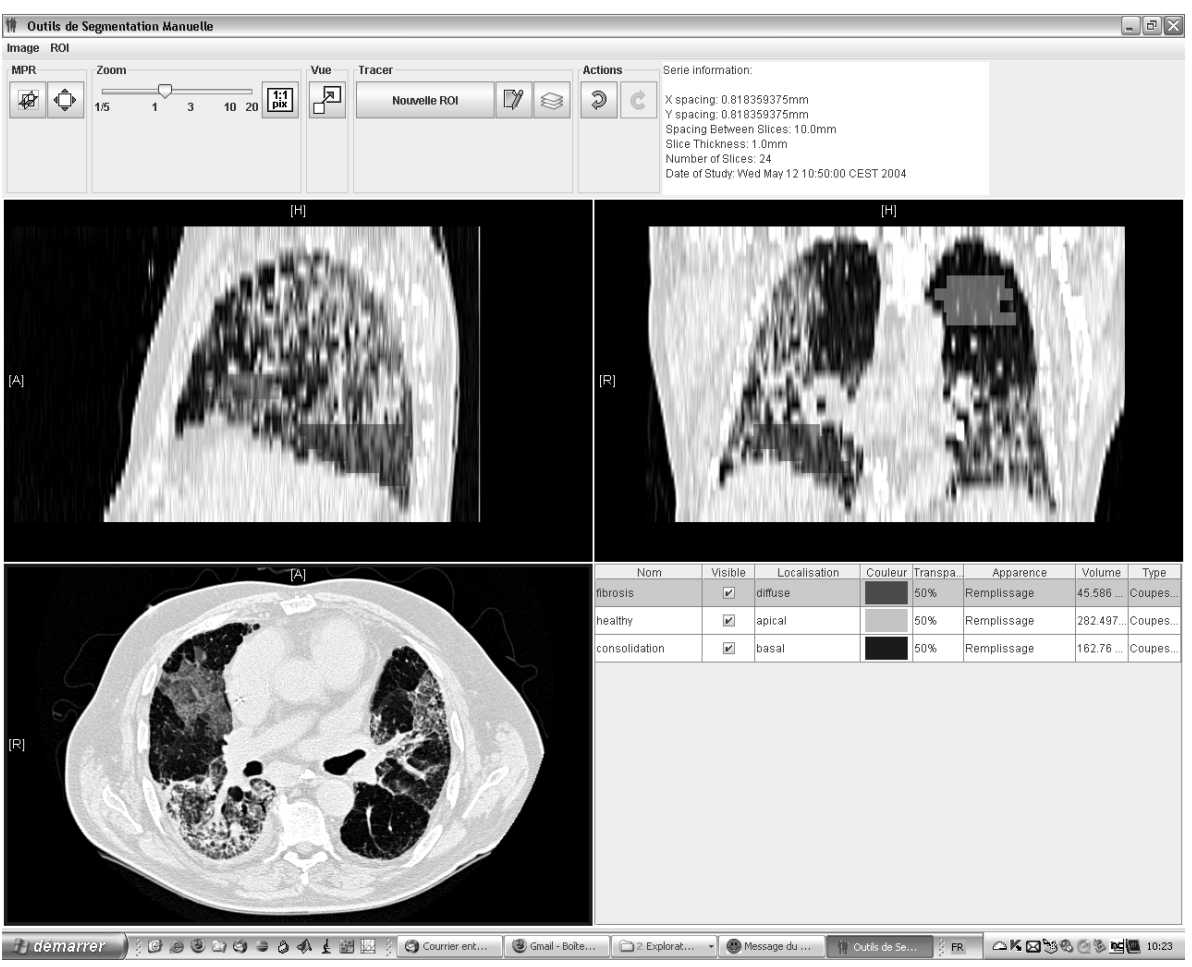

Fig. 2 A web interface to highlight regions in $3 \mathrm{D}$ image data to allow for effective tissue annotation, tissue classification, and search for similar cases.

aging has been hindering progress for a long time. Initiatives to change this were begun in the United States [34] and in Europe [35] to create a climate fostering image sharing and data exchange for the entire medical imaging domain. The NIH (National Institutes of Health) now also requires funded research projects to develop a strategy for making the data sets available for the long term. This has the potential to limit the need for expensive re-creation of datasets for each new research project.

Initiatives such as MIRC $^{22}$ (Medical Imaging Resource Center) of the RSNA (Radiological Society of North America) created a standard to make radiological teaching files available on the web using a single interface and allowing querying many databases with

\footnotetext{
22 http://mirc.rsna.org/
}

a single query, thus sharing the available knowledge. Some of the databases that are available via MIRC are also used in the ImageCLEF ${ }^{23}$ medical image retrieval benchmark that makes available images and the metadata together with query tasks. A gold standard defining expected responses for a retrieval system, and a comparison of the performance of participating systems $[12,36]$ are also distributed.

Another very important step in making data available is the Goldminer ${ }^{24}$ search engine of the American Roentgen Ray Society (ARRS) that currently makes accessible over 175,000 images from 228 journals. These images cannot at the moment be searched by their visual content but only by structured data such as age or gender of the per-

\footnotetext{
23 http://www.imageclef.org/

$24 \mathrm{http} / /$ golminer.arrs.org/
} 
son or by free text. The added value of this collection on the other hand is enormous as it not only makes available images with a limited annotation as in teaching files, but peer-reviewed full cases from many radiology journals. This connection of data to images has actually been one of the most important changes in image retrieval over the past years. Images can be linked with their existing annotations that are either structured or partially structured [36,37]. Several approaches to this exist, for example a simple bag of words approach and separate visual and textual queries [37], or the extraction of terms (MeSH - Medical Subject Headings or UMLS Unified Medial Language System) [38]. Connections with external data sources have also been attempted such as with existing relational databases $[39,40]$, which can help to increase the knowledge about an existing case just as Web 2.0 technologies increase an application's value by connecting various existing data sources.

There is enormous potential from direct access to images in the scientific literature as physicians can directly compare a current case with similar existing cases in the peer-reviewed literature. Such applications have been proposed [41], but now need to be implemented and integrated with the clinical patient record and large knowledge archives of literature such as Goldminer. Basically all approaches currently published claim to be knowledge-driven $[42,43]$, and this will mean that links to existing knowledge sources such as ontologies or literature archives will be increasingly common and create added value for a field that has for a long time concentrated on pure image processing technologies, leaving the image completely out of its clinical context. Algorithmic advantages in extracting and analysing visual feature spaces and distance measures between entities in these feature spaces are of course still necessary and being supplied [44].

As the amount of data involved in integrating several knowledge sources and large image repositories increases considerably, there is also a need for new approaches to efficient data processing. One potentially important technology are grid networks that have been used for image retrieval over the past few years and could allow for complex processing of even extremely large amounts of data [45] to reach a retrieval quality and speed sufficient for routine clinical applications.

A step seemingly in the opposite direction is the concentration of visual analysis for extremely small and well defined tasks such as modality detection [46]. These tasks might not be necessary if all images have a correct DICOM header (which is most often not the case) but it can be invaluable if images are in non-DICOM format (as is the case on the web or in journal articles) or when headers contain errors for gaining basic knowledge about the images. These data can subsequently be combined with other functions to create powerful retrieval capabilities.

Another very important Web 2.0 technology is the community building, which is also proposed in radiology around images [47]. In image retrieval such community building has started around the ImageCLEFmed [12,36] medical image retrieval benchmark. ImageCLEF is part of CLEF ${ }^{25}$ (Cross Language Evaluation Forum), a platform for benchmarking information retrieval research. A medical task started in ImageCLEF in 2004 and has been held every year since, with the goal to combine visual and textual cues for retrieval based on realistic search tasks. Such tasks are developed from

\footnotetext{
25 http://www.clef-campaign.org/
}

surveys among medical professionals, web log files of medical media search engines, or Medline search logs [11]. Since 2004, the community has become larger every year and almost 50 research groups registered for one of the two medical tasks in 2007. A joint workshop once a year creates a platform to discuss techniques, advancements in performance, realistic and challenging tasks, and plans for the years to come. Several researchers have started to propose their own tasks and run them in this forum, and many publications have been based on the data sets because many research groups which would otherwise not have had access to medical databases, realistic query tasks and relevance judgements of medical professionals to evaluate their algorithms now do.

\section{Some Missing Components}

We can see that many of the first trends that are included in the Web 2.0 name have also had a significant influence on medical multimedia retrieval, such as web-technologies to build interfaces that are hard to distinguish from desktop applications using AJAX. The availability of knowledge sources free of charge has started in the medical imaging domain, from teaching files, now to medical publications including all their images.

Still, there are many differences and shortcomings, partly because it is a much smaller community and reaching a critical mass is neither easy nor certain.

Trust is often not as high as in other non-critical domains (such as pop music or soccer), since decisions can have serious healthcare consequences which justifies this reticence. Standards for ensuring the quality of image labels might be able to help with this, and when trust is established, increased information exchange is likely to result. Another problem is that many knowl- 
edge resources in the medical field are expensive and not freely available such as SNOMED (Systemized Nomenclature of Medical Terms) or MeSH (Medical Subject Headings). This means that linkage with existing applications and databases is often far from simple. Curation, maintenance of these repositories and updates for new developments and knowledge sources is expensive. However, making these knowledge bases freely available could have a strong impact for many medical domains requiring the combination of textual and visual data for retrieval of cases.

A major expensive missing component is the generation of high quality data including annotations of regions in images and large amounts of the clinical data record relevant to the regions and their pathology. This will require both considerable time from clinicians with the necessary knowledge, and novel evaluation and testing methods for assessing their quality, which is hard (and strongly dependent on ontological assumptions as well as specific methods used in imaging), as well as serious questions about the challenges of legally defining pathophysiologies according to visual standards.

Another missing component is the availability of APIs for image retrieval and image analysis applications. In the non-medical field visual search tools have been integrated into search engines (for example face detection in images) and image search by manual sketches exists in FlickR. In the medical multimedia retrieval field it might be necessary to develop small components that are available on the web via APIs (via service oriented architectures) and that can be used to link several systems creating larger applications. These small blocks could be extremely varied, from indexing images from an entire site to extremely focused applications such as modality detection from images or
MeSH term extraction from medical texts or web pages.

Other important topics to follow are clearly a better analysis and treatment of full 3D data that is increasingly necessary $[48,49]$, as well as the use of augmented reality [50] and applications using multiple modalities [51,52]. This information has been used for data visualization but only very rarely for information retrieval.

All these applications are in their infancy, and medical information analysis and retrieval will undoubtedly continue to influence how the medical multimedia information retrieval field develops. In the end the multimedia retrieval field can link all the available information and help discover new required information just like a web search engine can do when we search for information on the Internet.

\section{Conclusions}

This article provides a short introduction to medical multimedia information retrieval and to Web 2.0 technologies. A review of the recent medical imaging and medical multimedia retrieval literature followed, highlighting the influence of Web 2.0 technologies on the medical visual information retrieval field. In many other fields consumer technologies also increasingly influence the business fields. It can be seen that many of the concepts of Web 2.0 have had influence on the medical multimedia field and this is likely to continue. Finally, many Web 2.0 applications influence the (gratis) participation of people in particular communities. In the medical field this is likely to take off in a similar way as several of the examples mentioned in the text already show. It seems particularly necessary to create simple and sharable building blocks with clear APIs, so application development can take advantage of not only data but also functionalities that already exist. This is in a way similar to Google earth that is now included in many applications, or other similar technologies.

The combination of knowledge bases and image datasets is underway. In the bioinformatics field this happened in a similar way with many databases becoming available free of charge (sometimes only for academia) or at nominal charges. Maintaining and then combining these data to generate new knowledge is currently an important work in this field and it could develop also in the medical field as more data actually become available. It seems important to have the databases and ontologies accessible free of charge or at moderate charges, so they can realize their full potential.

Content-based image or multimedia retrieval has the potential to become a building block in the Web 2.0 field as it can help connect the many datasets that are available, and that often contain some form of visual data. Creating such additional value by discovering knowledge hidden in the visual data could make medical multimedia analysis an everyday tool, albeit not always visible in the final applications. Currently, there is unfortunately a serious gap between the technology available in professional applications bought by and used in hospitals and research prototypes. Most research prototypes are only validated in a single clinical study and then often not used. Nodule detection in chest $\mathrm{CT}$ images is a notable exception with many vendors now offering solutions [53]. Still, this technology transfer needs to happen quicker to really take advantage of the field and make the technology available in clinical practice quickly, although some basic research is definitely necessary such as in brain-com- 
puter interfaces [54], where applications might still be several years away. Even consumer products often include new technology earlier and cheaper than clinical applications, for example to change the visualization of images (luminosity, level/window settings, segmentation), but also for information retrieval.

Of course, the medical domain has many peculiarities that need to be taken into account. Medical imaging equipment changes increasingly quickly. Machines are replaced by newer better ones that create high-resolution images. Protocols are changing towards tomographic images with smaller slice thicknesses, contrast agents evolve with images having higher contrast, and analogue equipment is changed to fully digital acquisition [55], and so on and so forth. Medical imaging evolves and changes quickly. Every reference data set will be outdated relatively quickly and for this reason it is important to develop flexible tools and update the databases and techniques quickly and have multimedia management tools that can be adapted to the varying conditions, for example in different countries (computed tomography protocols in one country are usually quite different from those in other countries, making standardization a key challenge for the future). Just like the Web, multimedia information analysis and retrieval is an extremely active and dynamic field that is currently in a state of constant change. It will most likely continue like this for the foreseeable future, given the strong and productive nature of medical imaging research.

\section{Acknowledgements}

This work was partially funded by the European Union through the KnowARC project (grant 032691), the Swiss National Science Foundation (FNS, grant 205321-109304/1), and the HES SO through the BeMeVIS project.

We thank the reviewers for their constructive comments for improving the paper.

\section{References}

1. Müller H, Michoux N, Bandon D, Geissbuhler A. A review of content-based image retrieval systems in medicine - clinical benefits and future directions. Int J Med Inform 2004:73:1-23.

2. Smeulders AWM, Worring M, Santini S, Gupta A, Jain R. Content-based image retrieval: The end of the early years. IEEE Transactions on Pattern Analysis and Machine Intelligence. 2000;22(12):1349-80.

3. Hirata K, Kato T. Query by visual example - Content-based image retrieval, International Conference on extending database technology. Springer Lecture Notes in Computer Science 580, 1992:23-7.

4. Lowe H, Antipov I, Hersh W, Arnott Smith C. Towards Knowledge-Based Retrieval of Medical Images. The role of semantic indexing, Image Content Representation and Knowledge-Based Retrieval. Annual Symposium of the American Society for Medical Informatics (AMIA); 1998. p. 882-6.

5. Tagare HD, Jaffe CC, Duncan J. Medical Image Databases, a content-based retrieval approach. JAm Med InformAssoc 1997;4:184-98.

6. Nitrosi A, Borasi G, Nicoli F, Modigliani G, Botti A, Bertolini M, Notari P. A filmless radiology department in a full digital regional hospital: quantitative evaluation of the increased quality and efficiency. J Digital Imaging 2007;20(2):140-8.

7. Aisen AM, Broderick LS, Winer-Muram H, Brodley CE, Kak AC, Pavlopoulou C, et al. Automated storage and retrieval of thin-section CT images to assist diagnosis: System description and preliminary assessment. Radiology 2003;228:265-70.

8. Lehmann TM, Plodowsky B, Spitzer K, Wein BB, Ney H, Seidl T. Extended Query refinement for content-based access to large medical image databases. SPIE Symposium on Medical Imaging; 2004. p. 5371(15):90-8.

9. Müller H, Lovis C, Geissbuhler A. Medical Image retrieval - the MedGIFT project. Medical Imaging and telemedicine 2005:2-7.

10. Shyu CR, Broadley CE, Kak AC, Broderick LS. ASSERT: A physician-in-the-loop content-based retrieval system for HRCT Image Databases. Computer Vision and image Understanding, 1999;75(12):111-32.

11. Müller H, Boyer C, Gaudinat A, Hersh W, Geissbuhler A. Analyzing Web Log Files of the Health On the Net HONmedia Search Engine to Define Typical Image Search Tasks for Image Retrieval Evaluation. medinfo 2007, Brisbane, Australia; 2007.

12. Müller H, Deselaers T, Lehmann T, Clough P, Kim E, Hersh W. Overview of the ImageCLEFmed 2006 Medical Retrieval and Medical Annotation Tasks. Springer Lecture Notes in Computer Science (LNCS); 2007.

13. Lessmann B, Nattkemper TW, Hans VH, Degenhard A. A method for linking computed image features to histological semantics in neuropathology, J Biomed Inform 2007:5.

14. Depeursinge A, Iavindrasana J, Hidki A, Cohen G, Geissbuhler A, Platon A, et al. A classification framework for lung tissue categorization. SPIE Medical Imaging; 2008.

15. Müller H, Do Huang PA, Depeursinge A, Hoffmeyer
P, Stern R, Lovis C, Geissbuhler A. Content-based image retrieval from a database of fracture images. SPIE Medical Imaging 2007; Volume 6516, San Diego, CA, USA; 2007.

16. Haux R. Health information system: Past, present, and future. Int J Med Inform 2006;75:268-81.

17. Liu BJ, Law MY, Documet J, Gertych A. Imageassisted knowledge discovery and decision support in radiation therapy planning, Comput Med Imaging Graph 2007;31(4-5):311-21.

18. Yamamoto S, Horiuchi J, Sekiguchi J, Wada S, Komizu M, Yamaguchi T. Design of a DICOM image-based program for estimating patient exposure dose in computed tomography. Technol Health Care 2007;15(2):147-56.

19. Moreno RA, Furuie SS, A contextual medical image viewer. IEEE Trans Inf Technol Biomed 2007;11(5):583-92.

20. AMIA, Secondary Uses and re-uses of healthcare data: Taxonomy for policy formulation and planning. internal publication of AMIA, available from http://www.amia.org/.

21. Safran C, Bloomrosen M, Hammond WE, Labkoff $\mathrm{S}$, Markel-Fox S, Tang PC, et al. Toward a National Framework for the Secondary Use of Health Data: An American Medical Informatics Association White Paper. JAm Med Inform Assoc 2007;14: 1-9.

22. Manos D. Secondary data use could cover technology costs. www.healthcarefinancenews.com, 1.1.2007.

23. Lovis C, Spahni, S, Cassoni N, Geissbuhler A. Comprehensive management of the access to the electronic patient record, Int J Med Inform 2007;76(5-6):466-70.

24. O'Reilly T. What is Web 2.0? O Reilly Publishers; 2005.

25. Giles J. Internet Encyclopedias go head to head. Nature 2005;438(7070):900-1.

26. Boyer C, Baujard, Scherrer, JR. HONcode: a standard to improve the quality of medical/health information on the Internet and HONs $5^{\text {th }}$ survey on the Use of the the Internet for Medical and Health Purposes. World Congress for Biomedical Sciences; 1999.

27. Tang H, Hwee Kwoon Ng J. Googling for a diagnosis - use of Google as a diagnostic aid: Internetbased study. Br Med J 2006;333:1143-5.

28. Giustini D. How Web 2.0 is changing medicine. $\mathrm{Br}$ Med J 2006;333:1283-4.

29. Basky E. Introducing Web 2.0: Weblogs and podcasting for health librarians. Journal of the Canadian Health Librarian Association 2006;27:33-4.

30. Charette RN. Visualizing electronic health records with Google-earth for the body. IEEE Spectrum, 2008; January.

31. Tommasi T, Orabona F, Caputo B. CLEF2007 Image Annotation Task: An SVM-based cue integration approach. CLEF working notes, 2007.

32. Zhang J, Sun J, Stahl J. PACS and web-based image distribution and display, Comput Med Imaging Graph 2003;27:197-206.

33. Rosset A, Müller H, Martins M, Dfouni N, Vallée JP, Ratib O. Casimage project: a digital teaching file authoring environment. Journal of Thoracic Imaging 2004;19:103-8.

34. Vannier MW, Summers RM. Sharing Images. Radiology 2003;228:23-5.

35. Horsch A, Prinz M, Schneider S, Sipil O, Spinnler 
$\mathrm{K}$, Vallée JP, et al. Establishing an Internation Reference Image Database for research and development in medical image processing, Methods Inf Med 2004:43:409-12.

36. Hersh W, Müller H, Jensen J, Yang J, Gorman P, Ruch P. Advancing Biomedical Image Retrieval: Development and Analysis of a Test Collection. J Am Med Inform Assoc (JAMIA) 2006; September/ October:488-96.

37. Kalpathy-Cramer J, Hersh W. Medical Image Retrieval and Automatic Annotation: OHSU at ImageCLEF 2007, CLEF working notes; 2007.

38. Ruiz ME. Combining image features, case descriptions and UMLS concepts to improve retrieval of medical images. AMIA annual symposium 2006. p. 674-8.

39. Demner-Fushman D, Antani S, Thoma G. Automatically finding images for clinical decision support. IEEE conference on Data Mining; 2007.

40. Atmosukarto I. A unifying framework for combining content-based image retrieval with relational database queries for biomedical applications. Conference of the Society for Imaging Informatics in Medicine. Seattle; 2008.

41. Deserno TM, Antani S, Long R. Exploring access to scientific literature using content-based image retrieval. SPIE Medical Imaging; 2007.

42. Scott G, Shyu CR. Knowledge-driven multidimensional indexing structure for biomedical media database retrieval, IEEE Trans Inf Technol Biomed 2007 May;11(3):320-31.

43. Erdal S, Catalyurek UV, Payne PR, Saltz J, Kamal
J, Gurcan MN. A knowledge-anchored integrative image search and retrieval system. J Digit Imaging 2007.

44. Felipe JC, Traina C Jr, Traina AJ. A new family of distance functions for perceptual similary retrieval of medical images. J Digit Imaging 2008.

45. Costa Oliveira M, Cime W, Azevedo Marques PM. Towards applying content-based image retrieval in clinical routine. Future Generation computer systems 2007:23(3):466-74.

46. Kalpathy-Cramer J, Hersh W. Automatic image modality classification and annotation to improve medical image retrieval. Medinfo 2007;12(2):1334-8.

47. Kahn CE. Web 2.0 applications in Radiology: Feasibility of a dynamic, context-sensitive inline image gallery. Conference of the Society for Imaging Informatics in Medicine, Seattle; 2008.

48. Grau V, Becher H, Noble JA. Registration of multiview real-time 3-D echocardiographic sequences. IEEE Trans Med Imaging 2007;26(9): 1154-65.

49. Reyes Aldasoro CC, Bhalerao B. Volumetric texture segmentation by discriminant feature selection and multiresolution classification. IEEE Trans Med Imaging 2007;26(1):1-14.

50. Lovo EE, Quintana JC, Puebla MC, Torrealba G, Santos JL, Lira IH, et al. A novel, inexpensive method of image coregistration for applications in image-guided surgery using augmented reality. Neurosurgery, 2007;60(4 Suppl 2):366-71; discussion 371-2.

51. Reinertsen I, Lindseth F, Unsgaard G, Collins DL. Clinical validation of vessel-based registration for correction of brain-shift. Med Image Anal 2007;30.

52. Curiel L, Chopra R, Hyninen K. Progress in Multimodality Imaging: Truly simultaneous Ultrasound and Magnetic Resonance Imaging. IEEE Trans Med Imaging 2007;26(12):1740-6.

53. Saba L, Caddeo G, Mallarini G. Computer-aided detection of pulmonary nodules in computed tomography: analysis and review of the literature. Journal of Computer Assisted Tomography 2007; 31(4):611-9.

54. Friman O, Volosyak I, Graser A. Multiple channel detection of steady-state visual evoked potentials for brain-computer interfaces. IEEE Trans Biomed Eng 2007;54(4):742-50.

55. Snoeren PR, Karssemeijer N. Gray-scale and geometric registration of full-field digital and filmscreen mammograms. Med Image Anal 2007;11(2): 146-56.

\section{Correspondence to:}

Prof. Dr. Henning Müller

University \& Hospitals of Genevo

Medical Informatics and

University of Applied Sciences

Business Information Systems

TecnoARK 3

3960 Sierre, Switzerland

Tel: + 41276069012

Fax: + 41276069000

E-mail: henning.mueller@hevs.ch 